\title{
Perfil de risco de queda de idosos internados em uma unidade de internação geriátrica de um hospital universitário do município de Porto Alegre
}

\author{
Gabriela Guimarães Oliveira*, Mara Regina Knorst**, Clarissa Netto Blattner ${ }^{\star * *}$, \\ Janete de Souza Urbanetto ${ }^{\star \star \star \star}$, Raquel Milani El-Kik ${ }^{\star \star \star \star \star}$
}

\section{Resumo}

As quedas representam um importante problema de saúde para a população idosa, culminam em perda da autonomia funcional e, consequentemente, da qualidade de vida. Este estudo tem por objetivo observar o risco de queda de idosos internados em uma unidade de internação geriátrica assim como os fatores associados que influenciam tal evento. Trata-se de um estudo descritivo transversal. Além da avaliação do risco de queda, por intermédio de uma escala (Morse Fall Scale - MFS) e de um teste (Timed Up and Go - TUG), foi aplicado um instrumento composto por dados sociodemográficos (sexo e idade), questionamento sobre a prática de atividade física pré-hospitalar e a avaliação de fatores associados a quedas, como força muscular (Força de Preensão Palmar - FPP) e capacidade funcional (índice de Katz). O estudo acompanhou 30 idosos. A maior parte dos idosos era do sexo feminino $(70 \%)$, longevos $(56,7 \%)$ e sedentários
(73,3\%). De acordo com o TUG, a maior parte dos idosos encontrava-se em risco médio de queda (56,7\%). De acordo com a MFS, a maior parte dos idosos encontrava-se em risco elevado de queda $(80 \%)$. Seis pacientes $(19,8 \%)$ tiveram queda da própria altura. De acordo com o índice de Katz, a maior parte dos idosos apresentava maior funcionalidade (36,7\%). Também, a maior parte dos idosos apresentava FPP diminuída. De acordo com os resultados obtidos, pode-se concluir que o risco de queda mostrou-se de médio a elevado na população observada e que houve a prevalência do sexo feminino e de longevos, o que sugere um maior risco de queda em tal população. Observou-se, ainda, uma porcentagem grande de sedentários e de idosos com força muscular reduzida, o que pode estar associado ao perfil de risco de quedas dessa população.

Palavras-chave: Acidentes por quedas. Hospitalização. Idoso.

* Fisioterapeuta. Residente em Saúde do Idoso no Hospital São Lucas da Pontifícia Universidade Católica do Rio Grande do Sul. Porto Alegre, RS, Brasil. Endereço para a correspondência: Avenida Heitor Vieira, 1920, Belém Novo, Porto Alegre, RS, CEP: 91780-000. E-mail: oliveira_gabriela@hotmail.com

* Fisioterapeuta. Doutora em Gerontologia Biomédica Pontifícia Universidade Católica do Rio Grande do Sul. Professora da Faculdade de Enfermagem, Nutrição e Fisioterapia da Pontifícia Universidade Católica do Rio Grande do Sul. Porto Alegre, RS, Brasil. E-mail: mknorst@pucrs.br

*** Fisioterapeuta. Doutora em Cardiologia pela Universidade Federal do Rio Grande do Sul. Professora da Faculdade de Enfermagem, Nutrição e Fisioterapia da Pontifícia Universidade Católica do Rio Grande do Sul. Porto Alegre, RS, Brasil. E-mail: clarissa.blattner@pucrs.br

**** Enfermeira. Doutora em Ciências da Saúde pela Pontifícia Universidade Católica do Rio Grande do Sul. Professora da Faculdade de Enfermagem, Nutrição e Fisioterapia da Pontifícia Universidade Católica do Rio Grande do Sul. Porto Alegre, RS, Brasil. E-mail: jurbanetto@pucrs.br

****** Nutricionista. Doutoranda em Gerontologia Biomédica na Pontifícia Universidade Católica do Rio Grande do Sul. Professora da Faculdade de Enfermagem, Nutrição e Fisioterapia da Pontifícia Universidade Católica do Rio Grande do Sul. Porto Alegre, RS, Brasil. E-mail: raquel.elkik@pucrs.br

$\rightarrow$ http://dx.doi.org/10.5335/rbceh.v13i2.5945

Recebido em: 22.04.2016. Aceito em: 09.11.2016. 


\section{Introdução}

Atualmente, no Brasil, o envelhecimento acontece de forma acelerada. O ritmo de crescimento da população idosa tem sido sistemático e consistente. Segundo o censo demográfico de 2010, a população brasileira compreende 190.755.199 milhões de pessoas. $\mathrm{O}$ contingente de pessoas idosas ( $\geq 60$ anos) é de 20.590 .599 milhões, ou seja, aproximadamente, $10,8 \%$ da população total. Projeções indicam que, em 2020, a população idosa brasileira será de 30,9 milhões, representando $14 \%$ da população total (INSTITUTO BRASILEIRO DE GEOGRAFIA E ESTATÍSTICA, 2010).

Nesse contexto, o envelhecimento é uma temática que é estudada constantemente. $\mathrm{O}$ processo de envelhecimento manifesta-se pelo declínio das funções dos órgãos e sistemas, o que faz com que o organismo comece a apresentar falhas diante das funções de manutenção corporal. O comprometimento da manutenção corporal gera a instabilidade postural, que vem acompanhada da dificuldade em manter posturas adequadas, ocasionando, frequentemente, as quedas, um dos principais fatores que limitam a vida do idoso hoje (BECHARA; SANTOS, 2008).

A queda é considerada um deslocamento não intencional do corpo para um nível inferior à posição inicial, sem correção em tempo hábil, determinada por circunstâncias multifatoriais que comprometem a estabilidade postural. Ela ocupa o segundo lugar na frequência de internações e gastos para a saúde pública. Aponta-se que cerca de $30 \%$ dos idosos com idade igual ou superior a 65 anos caem pelo menos uma vez ao ano, e essa proporção aumenta para $50 \%$ nos idosos acima dos 80 anos (SIQUEIRA, 2007).

As quedas representam um importante problema de saúde para a população idosa, pois culminam em perda da autonomia funcional e da qualidade de vida. Entre os principais fatores de risco, encontram-se histórico de múltiplas quedas, alterações do equilíbrio postural, diminuição da força muscular decorrente da sarcopenia, presença de comorbidades e uso de determinados medicamentos (NASCIMENTO et al., 2009).

Durante o período de internação hospitalar, os idosos tornam-se mais suscetíveis aos eventos adversos da hospitalização, tais como restrição ao leito e perda de massa óssea e muscular, debilidade física, polifarmácia e procedimentos terapêuticos, alterações nutricionais, privação sensorial e comorbidades agudas ou crônicas descompensadas. Tais complicações podem levar a um declínio funcional e, consequentemente, a uma maior instabilidade postural (SALES et al., 2010).

De modo geral, a hospitalização aumenta o risco de queda. A literatura indica que a taxa de queda de pacientes em hospitais de países desenvolvidos varia de 3 a 5 quedas por 1.000 pacientes/ dia. Aponta-se, também, que as quedas não se distribuem uniformemente nos hospitais, sendo mais frequentes nas unidades com concentração de pacientes idosos (OLIVER; HEALEY; HAINES, 2010; DYKES et al., 2010). 
Desse modo, estudar fatores associados ao risco de queda em idosos tornou-se um grande desafio para a comunidade científica. Portanto, sendo o processo de envelhecimento um acontecimento inevitável, acompanhado de declínio funcional e alterações de equilíbrio, este estudo tem por objetivo traçar o perfil de idosos com risco de queda internados em uma unidade de internação geriátrica de um hospital universitário do município de Porto Alegre, com a utilização de instrumentos e escalas de avaliação de risco.

\section{Materiais e métodos}

Trata-se de um estudo descritivo transversal, realizado em um hospital universitário da região metropolitana de Porto Alegre, Rio Grande do Sul, Brasil, durante o período de maio a setembro de 2015.

A amostra do estudo foi composta de idosos ( $\geq 60$ anos) hospitalizados em uma unidade de internação geriátrica. Foram incluídos idosos que puderam ser avaliados, preferencialmente, em $24 \mathrm{~h}$ ou até $48 \mathrm{~h}$ da data de internação. Aqueles em que não foi possível aplicar pelo menos um dos testes ou escalas foram excluídos do estudo.

A coleta de dados foi realizada por uma equipe capacitada e devidamente treinada, com a orientação de um manual orientador, elaborado pelos próprios pesquisadores. Foi realizada uma única avaliação em cada paciente incluído na pesquisa.

Além da avaliação do risco de queda, por meio de uma escala e um teste, o instrumento era composto por dados sociodemográficos (sexo e idade), questionamento sobre a prática de atividade física pré-hospitalar e a avaliação de fatores associados a quedas, como força muscular e capacidade funcional. Em relação à prática de atividade física, considerou-se o que é proposto pela Organização Mundial da Saúde (2010), que orienta que o ideal para indivíduos idosos é fazer pelo menos 150 minutos de atividade física aeróbica de intensidade moderada durante a semana ou fazer pelo menos 75 minutos de atividade física aeróbica de intensidade vigorosa durante a semana.

A força muscular (FM) foi avaliada pela aferição da força de preensão palmar (FPP), que foi obtida pelo dinamômetro hidráulico manual JAMAR®, com ajuste na segunda posição da empunhadura. Nessa avaliação, o indivíduo é posicionado sentado em uma cadeira com encosto, com as costas apoiadas, o ombro em adução, cotovelo fletido a $90^{\circ}$ e antebraço em semipronação. Durante a preensão palmar, o braço permanece imóvel, sem apoio, e, havendo somente a flexão das articulações interfalangeanas e metacarpofalangeanas, são realizadas três medições em cada mão, sendo considerada a de maior valor (BOHANNON, 2006; DIAS, 2010; MACEDO; FREITAS; SCHEICHER, 2014). Cruz-Jentoft et al. (2010) recomendam a FPP como medida de rastreamento de sarcopenia e risco de limitação de mobilidade em idosos e definem os pontos de corte de 30kgf para homens e $20 \mathrm{kgf}$ para mulheres. Sendo assim, sua avaliação é considerada um 
importante marcador de fragilidade no idoso, como também um instrumento de avaliação de risco de queda, pois se trata de um potente indicador de força muscular global.

A capacidade funcional ( $C F$ ) foi avaliada pelo índice de Katz. Esse instrumento tem como finalidade avaliar o padrão de independência para a realização das atividades de vida diária (AVDs) (DUARTE; ANDRADE; LEBRÃO, 2007). $\mathrm{O}$ índice de Katz é composto por seis itens avaliativos: banho, vestir, ir ao banheiro, transferência, continência e alimentação. Neste estudo, utilizamos a versão original do índice (KATZ et al., 1963). A classificação funcional final utilizada foi a modificada por Rubenstein et al. (1984), que classifica em mais funcional, funcionalidade intermediária e menos funcional. A avaliação da capacidade funcional pelo índice de Katz está intimamente relacionada com o risco de queda, ao passo que o baixo desempenho funcional altera o controle postural, gerando instabilidade e pondo em risco a movimentação segura dos idosos, predispondo tal evento (MACIEL; GUERRA, 2007).

$\mathrm{O}$ risco de queda foi avaliado pela Morse Fall Scale (MFS), na versão em língua portuguesa, e pelo Timed Up and Go (TUG). A MFS é uma escala criada para o monitoramento do risco de queda no ambiente hospitalar. É uma escala traduzida e adaptada para a língua portuguesa que apresenta excelente reprodutibilidade. Além disso, é de fácil aplicabilidade e destaca-se pela aparente simplicidade de seus itens de avaliação, que são: histórico de queda; diagnóstico secundário; auxílio na deambulação; terapia endovenosa/dispositivo endovenoso salinizado ou heparinizado; marcha; estado mental. A soma das pontuações de cada item gera um escore para a classificação do risco em baixo ( 0 - 24 pontos), moderado ( 25 - 44 pontos) e elevado ( $\geq 45$ pontos) (MORSE; MORSE; TYLKO, 1989; URBANETTO et al., 2013).

O TUG é um teste que quantifica o desempenho da mobilidade por meio da velocidade ao realizar uma tarefa. $\mathrm{O}$ teste avalia o equilíbrio e a mobilidade funcional em pequenas tarefas motoras essenciais para uma vida independente, como o autocontrole postural e o equilíbrio, apresentando uma boa correlação com medidas relacionadas às quedas e à instabilidade. $\mathrm{O}$ teste consiste em levantar-se de uma cadeira com encosto, sem ajuda dos braços, andar a uma distância de três metros, dar a volta e retornar. No início do teste, o paciente deve estar com o dorso apoiado no encosto da cadeira e, ao final, deve encostar novamente. $\mathrm{O}$ paciente deve receber a instrução "vá" para realizar o teste e o tempo será cronometrado em segundos a partir da voz de comando até o momento em que ele apoie novamente o dorso no encosto da cadeira. $O$ teste deve ser realizado uma vez para familiarização e uma segunda vez para tomada do tempo. O tempo gasto para realizar o teste gera uma classificação do risco em baixo $(<10$ segundos), médio (10 - 20 segundos) e elevado (> 20 segundos) (LIN et al., 2004; PODSIADLO; RICHARDSON, 1991). 
Foram avaliados 63 idosos, mas somente 30 foram incluídos neste estudo. Assim, 33 foram excluídos, pois não conseguiram realizar o TUG e/ou a FPP.

Poucos foram os idosos que apresentaram dificuldade na execução ou no entendimento dos testes/escalas. Quando essa dificuldade se apresentava e o teste/escala permitia tal intervenção, foi necessária a participação de familiares/ cuidadores na coleta de dados.

Os dados foram inseridos em um banco de dados no programa Microsoft Excel. A análise foi realizada no Statistical Package for the Social Sciences (SPSS 17.0), utilizando a estatística descritiva (frequência absoluta e relativa e de variabilidade).

O projeto de pesquisa foi aprovado pelo Comitê de Ética em Pesquisa da PUCRS (OF. CEP - 1272/09) e com o Parecer 636.335. Os participantes ou os responsáveis assinaram o termo de consentimento livre e esclarecido.

\section{Resultados}

Neste estudo, a média de idade encontrada foi 80,70 anos, sendo o mínimo 65 anos e o máximo 101 anos. Houve uma prevalência do sexo feminino (70\%) e a maioria dos idosos eram longevos $(56,7 \%)$ e sedentários $(73,3 \%)$ (Tabela 1$)$.

Tabela 1 - Caracterização da amostra

\begin{tabular}{|c|c|}
\hline Variáveis & $\mathrm{n}=\mathbf{3 0}(\%)$ \\
\hline Idade - Média \pm DP [mín - máx] & $80,70 \pm 8,305[65-101]$ \\
\hline \multicolumn{2}{|l|}{ Idade (anos) } \\
\hline 60 a 79 & $13(43,3)$ \\
\hline$\geq 80$ & $17(56,7)$ \\
\hline \multicolumn{2}{|l|}{ Sexo } \\
\hline Masculino & $9(30)$ \\
\hline Feminino & $21(70)$ \\
\hline \multicolumn{2}{|l|}{ Atividade física } \\
\hline Sim & $8(26,7)$ \\
\hline Não & $22(73,3)$ \\
\hline
\end{tabular}

Fonte: Dados da pesquisa.

Nota: idade em anos; $\mathrm{DP}=$ desvio-padrão; mín. = mínimo; máx. = máximo; $\mathrm{n}=$ número de indivíduo; $\%$ = porcentagem.

De acordo com o TUG, a maioria dos idosos encontrava-se em risco médio de queda $(56,7 \%)$. A média na realização do teste foi de 22,47 segundos, sendo 8 segundos o mínimo e 124 segundos o máximo (Tabela 2). 
Tabela 2 - Timed Up and Go

\begin{tabular}{c|c}
\hline \multicolumn{1}{c|}{ TUG } & $\mathbf{n}=\mathbf{3 0}(\%)$ \\
\hline TUG (s) - Média \pm DP [mín. - máx.] & $22,47 \pm 20,529[8-124]$ \\
Classificação & $2(6,7)$ \\
Risco baixo & $17(56,7)$ \\
Risco médio & $11(36,6)$ \\
Risco elevado & \\
\hline
\end{tabular}

Fonte: Dados da pesquisa.

Nota: idade em anos; $\mathrm{DP}=$ desvio-padrão; mín. = mínimo; máx. = máximo; $\mathrm{n}$ = número de indivíduo; $\%$ = porcentagem.

De acordo com a MFS, a maioria dos idosos encontravam-se em risco elevado de queda (80\%). De acordo com os itens avaliados pela MFS, a maioria dos idosos relataram histórico de queda nos últimos três meses $(60 \%)$, apresentavam mais de um diagnóstico $(96,7 \%)$, não utilizavam auxílio na deambulação ou eram total- mente acamados ou deambulavam com auxílio (80\%), utilizavam dispositivo endovenoso $(83,3 \%)$, apresentavam a marcha fraca $(53,3 \%)$ e eram orientados/capazes quanto à capacidade e à limitação (83,3\%). Apenas um indivíduo encontrava-se em risco baixo de queda $(3,3 \%)$ (Tabela 3$)$.

Tabela 3 - Morse Fall Scale

\begin{tabular}{|c|c|}
\hline MSF & $n=30(\%)$ \\
\hline \multicolumn{2}{|l|}{ Classificação } \\
\hline Risco baixo & $1(3,3)$ \\
\hline Risco médio & $5(16,7)$ \\
\hline Risco elevado & $24(80,0)$ \\
\hline \multicolumn{2}{|l|}{ Histórico de queda } \\
\hline Não & $12(40,0)$ \\
\hline Sim & $18(60,0)$ \\
\hline \multicolumn{2}{|l|}{ Diagnóstico secundário } \\
\hline Não & $1(3,3)$ \\
\hline Sim & $29(96,7)$ \\
\hline \multicolumn{2}{|l|}{ Auxílio na deambulação } \\
\hline Não utiliza & $24(80)$ \\
\hline \multicolumn{2}{|l|}{ Totalmente acamado } \\
\hline \multicolumn{2}{|l|}{ Deambula com auxílio } \\
\hline Usa muleta/bengala/andador & $6(20)$ \\
\hline Segura-se no mobiliário/parede & $0(0,00)$ \\
\hline \multicolumn{2}{|l|}{ Dispositivo endovenoso } \\
\hline Não & $5(16,7)$ \\
\hline Sim & $25(83,3)$ \\
\hline \multicolumn{2}{|l|}{ Marcha } \\
\hline Normal & $7(23,3)$ \\
\hline \multicolumn{2}{|l|}{ Não deambula/totalmente acamado } \\
\hline \multicolumn{2}{|l|}{ Usa cadeira de rodas } \\
\hline Marcha fraca & $16(53,3)$ \\
\hline Marcha comprometida/cambaleante & $7(23,3)$ \\
\hline \multicolumn{2}{|l|}{ Estado mental } \\
\hline Orientado & $25(83,3)$ \\
\hline Superestima & $5(16,7)$ \\
\hline
\end{tabular}

Fonte: Dados da pesquisa.

Nota: $\mathrm{n}=$ número de indivíduo; $\%=$ porcentagem. 
Neste estudo, os idosos da pesquisa foram monitorados durante a internação quanto à ocorrência de quedas. Assim, seis pacientes $(19,8 \%)$ tiveram queda da própria altura, que foram detectadas pela equipe de enfermagem, porém, sem danos físicos. Todas as quedas ocorreram no banheiro. Os idosos que caíram durante a internação tiveram dano psicológico, sendo o medo de cair novamente a principal consequência.

Na população em estudo, o resultado obtido pelo TUG e pela MFS não foram idênticos, pois o TUG indicou a preva- lência de risco médio $(56,7 \%)$ e a MFS a prevalência de risco elevado (80\%).

De acordo com o índice de Katz, a maioria dos idosos apresentavam maior funcionalidade $(36,7 \%)$. De acordo com os itens avaliados pelo índice de Katz, a maioria tinha dependência parcial nas atividades de banho (40\%), vestir (53,3\%) e continência (50\%) e independência nas atividades de ir ao banheiro (56,7\%), transferência $(63,3 \%)$ e alimentação (70\%). Em nenhum item avaliado houve a maior prevalência de dependência total (Tabela 4).

Tabela 4 - Índice de Katz

\begin{tabular}{|c|c|}
\hline Índice de Katz & $n=30(\%)$ \\
\hline $\begin{array}{l}\text { Classificação } \\
\text { Maior funcionalidade } \\
\text { Funcionalidade intermediária } \\
\text { Menor funcionalidade }\end{array}$ & $\begin{array}{c}11(36,7) \\
9(30,0) \\
10(33,3)\end{array}$ \\
\hline $\begin{array}{l}\text { Banho } \\
\text { Dependência completa } \\
\text { Dependência parcial } \\
\text { Independência }\end{array}$ & $\begin{array}{c}7(23,3) \\
12(40) \\
11(36,7)\end{array}$ \\
\hline $\begin{array}{l}\text { Dependência completa } \\
\text { Dependência parcial } \\
\text { Independência }\end{array}$ & $\begin{array}{c}5(16,7) \\
16(53,3) \\
9(30,0)\end{array}$ \\
\hline $\begin{array}{l}\text { Ir ao banheiro } \\
\text { Dependência completa } \\
\text { Dependência parcial } \\
\text { Independência }\end{array}$ & $\begin{array}{c}2(6,7) \\
11(36,7) \\
17(56,7)\end{array}$ \\
\hline $\begin{array}{l}\text { Transferência } \\
\text { Dependência completa } \\
\text { Dependência parcial } \\
\text { Independência }\end{array}$ & $\begin{array}{c}1(3,3) \\
10(33,3) \\
19(63,3)\end{array}$ \\
\hline $\begin{array}{l}\text { Continência } \\
\text { Dependência completa } \\
\text { Dependência parcial } \\
\text { Independência }\end{array}$ & $\begin{array}{c}1(3,3) \\
15(50,0) \\
14(46,7)\end{array}$ \\
\hline $\begin{array}{l}\text { Alimentação } \\
\text { Dependência completa } \\
\text { Dependência parcial } \\
\text { Independência }\end{array}$ & $\begin{array}{c}2(6,7) \\
7(23,3) \\
21(70,0)\end{array}$ \\
\hline
\end{tabular}

Fonte: Dados da pesquisa.

Nota: $\mathrm{n}=$ número de indivíduo; \% = porcentagem. 
De acordo com a FPP a média da mão dominante foi de 17,33 kgf, sendo o mínimo 6 kgf e o máximo 42 kgf. Já a média da mão não dominante foi de 16,67 kgf, sendo o mínimo 4 kgf e o máximo 40 kgf. A maioria dos idosos apresentavam FPP diminuída, tanto para a mão dominante $(80 \%)$ como para a não dominante $(83,3 \%)$ (Tabela 5).

Tabela 5 - Força de Preensão Palmar

\begin{tabular}{c|c}
\hline FPP & $\mathbf{n = 3 0}(\%)$ \\
\hline Mão dominante & $17,33( \pm 8,108)[6-42]$ \\
FPP (Kgf) - Média \pm DP [mín. - máx.] & $24(80,0)$ \\
FPP diminuída & $6(20,0)$ \\
FPP adequada & \\
Mão não dominante & $16,67( \pm 7,884)[4-40]$ \\
FPP (Kgf) - Média \pm DP [mín. - máx.] & $25(83,3)$ \\
FPP diminuída & $5(16,7)$ \\
FPP adequada &
\end{tabular}

Fonte: Dados da pesquisa.

Nota: idade em anos; $\mathrm{DP}=$ desvio-padrão; mín. = mínimo; máx. = máximo; $\mathrm{n}$ = número de indivíduo; $\%$ = porcentagem .

\section{Discussão}

Neste estudo, a média de idade encontrada foi 80,70 anos, com um predomínio de mais de $50 \%$ de pacientes do sexo feminino, o que vai ao encontro do estudo de Welfer (2007), que, ao pesquisar as causas de internação de idosos em uma unidade geriátrica do mesmo município, encontrou uma média de idade de 80,0 anos, com um predomínio de $58 \%$ de pacientes do sexo feminino.

Da mesma forma, um estudo internacional encontrou nas mulheres uma probabilidade de risco para quedas de 1,94 vezes maior do que em homens, apontando que tal prevalência possa estar relacionada ao melhor estado funcional das mulheres idosas em comparação aos homens idosos, favorecendo assim um maior risco de queda (LIN et al., 2011). Para Alvares et al. (2010), o fato de as mulheres envolverem-se mais em atividades domésticas parece ser outro fator que as deixaria mais susceptíveis a tal evento. Contudo, de acordo com Rodrigues, Fraga e Barros (2014), o fato de as mulheres apresentarem menor quantidade de massa magra e de força muscular pode ser também um fator que as exponha mais a tal risco.

Segundo Bós e Kimura (2015), os longevos internados em unidades geriátricas apresentam maior frequência de hospitalizações por condições sensíveis à atenção primária (CSAP), ou seja, por um conjunto de problemas de saúde para os quais a efetiva ação da atenção primária diminuiria o risco de internações, o que explica a prevalência dessa população neste estudo. Além disso, a prevalência do sexo feminino pode ser explicada por Camargos, Perpétuo e Machado (2005), que apontam que as 
mulheres idosas paulistanas apresentam maior expectativa de vida do que os homens, no entanto, uma maior proporção de anos vividos, com mais comorbidades.

Guimarães et al. (2004) compararam a propensão de queda entre idosos que praticavam atividade física e idosos sedentários. Os autores encontraram que, de acordo com o TUG, os idosos praticantes tinham risco baixo de queda e os não praticantes tinham risco médio. Tais achados são compatíveis com este estudo, em que grande parcela dos idosos relataram inatividade física antes da internação hospitalar e encontravam-se em médio risco de queda, de acordo com o TUG. Tal fato sugere que indivíduos sedentários apresentam menor mobilidade e maior propensão a quedas quando comparados àqueles que praticam atividade física regularmente. Sendo assim, a prática de atividade física regular é uma forma de prevenir quedas em pessoas idosas.

A classificação dos pacientes conforme a MFS apontou que a maioria dos idosos encontravam-se em risco elevado de queda. Esses resultados corroboram os achados encontrados por Al Tehewy, Amin e Nassar (2015), que realizaram um estudo longitudinal com 411 pacientes idosos internados e identificaram uma maior prevalência de idosos com risco elevado de queda.

Evans et al. (2001) realizaram uma revisão sistemática acerca dos fatores de risco para queda em ambiente hospitalar e relataram como um dos fatores mais frequentes a história de queda prévia, acreditando-se que de $16 \%$ a $52 \%$ dos pacientes com esse relato apresentarão um novo episódio durante o período de internação. Fato que vai ao encontro dos achados deste estudo, em que mais de a metade dos idosos referiram queda recente.

Ferreira e Yoshitome (2010), ao avaliar a prevalência e as caraterísticas das quedas de idosos institucionalizados, observaram que idosos portadores de diversas patologias eram mais suscetíveis a tal evento. No presente estudo, quase $100 \%$ dos idosos apresentaram mais de um diagnóstico.

A maioria dos idosos da amostra não utilizavam auxílio ou eram acamados ou, ainda, deambulam com auxílio de profissionais da saúde, pontuando nesse item da MFS um escore baixo. Para Urbanetto et al. (2013), o paciente enquadrado nessa classificação apresenta menor risco de queda do que aquele que usa muleta/ bengala/andador ou sustenta-se no mobiliário/parede. Assim, acredita-se que o maior percentual nessa classificação tenha ocorrido pelo fato de se tratar de uma unidade de internação geriátrica em que os profissionais técnicos são orientados a assegurar o deslocamento seguro dos pacientes, mesmo que eles utilizem dispositivos de marcha, assim como pelo fato de os pacientes, muitas vezes, não terem à sua disposição, no ambiente hospitalar, os dispositivos que utilizam em casa ou na instituição.

Neste estudo, grande porcentagem dos idosos utilizavam dispositivo endovenoso, fator que, para Dykes et al. (2009), é considerado de risco para ocorrência de quedas, ao passo que o paciente dirige 
uma atenção especial a ele, o que pode distraí-lo durante a mobilização/deambulação e colocá-lo em situação de risco. Abreu et al. (2015) avaliaram a incidência e os fatores preditores de queda em idosos hospitalizados, observando que piores condições de equilíbrio e marcha são consideradas variáveis preditoras para esse evento. Acredita-se que isso ocorra devido às modificações que a marcha sofre com o envelhecimento, tornando os passos mais curtos, mais lentos e menos seguros, predispondo as quedas. No presente estudo, a maioria dos idosos apresentaram marcha fraca e, possivelmente, encontravam-se em maiores condições de risco.

De acordo com Reis et al. (2014), a alteração do estado mental é o fator de risco de queda mais comumente identificado e, provavelmente, o mais difícil de manejar em termos de diminuição do risco. No entanto, neste estudo, poucos idosos apresentaram alteração do estado mental.

Ainda, apenas um indivíduo encontrava-se em risco baixo de queda. Sugere-se que tal fato tenha ocorrido porque a amostra consistia, em maior parte, em idosos longevos e, de acordo com Brito et al. (2013), a prevalência de quedas nos idosos longevos é maior.

A maior parte dos idosos da amostra encontrava-se em risco elevado de queda, de acordo com a MFS, e apenas seis pacientes tiveram queda da própria altura durante o período de internação. Sugerimos que tal fato possa ter ocorrido devido à inexistência de um instrumento adequado de notificação de quedas no ambiente hospitalar, além do déficit de conhecimento dos profissionais em relação ao conceito de queda e do medo dos profissionais de enfermagem acerca da punição pela ocorrência desse evento, estimulando assim a subnotificação (DUARTE et al., 2015).

Os idosos que caíram apresentaram dano psicológico relacionado ao evento, relatando o medo de cair novamente. De acordo com Ishizuka et al. (2005), o medo de cair pode ser um fator protetor quando o idoso toma mais cuidado para não se expor ao risco, mas também pode ser um fator negativo quando causa limitação e insegurança, mostrando-se um importante preditor de risco aumentado de queda. Assim como neste estudo, ao estudar a ocorrência e fatores associados a quedas em idosos institucionalizados, Lojudice et al. (2010) encontrou que, em relação aos fatores extrínsecos, o local de maior ocorrência de queda é o banheiro.

Neste estudo, o TUG e a MFS não avaliaram o risco de queda da mesma forma, apresentando, assim, uma classificação diferente entre os instrumentos de avaliação. Sugere-se que tal fato tenha ocorrido devido ao fato de a amostra ser pequena, devido às diversas variações de ponto de corte que o TUG apresenta ou pelo TUG não ser um teste adequado para a realidade hospitalar, pois, embora disponha de uma avaliação do equilíbrio geral, não possibilita o discernimento preciso de déficits estáticos ou dinâmicos (SHUBERT, 2006). Ao passo que, de outro modo, a MFS é capaz de prever pacientes com risco de queda e identificar mais de $80 \%$ das quedas, 
provando ser uma escala ideal e bastante específica para o ambiente hospitalar (AL TEHEWY; AMIN; NASSAR, 2015).

De acordo com o índice de Katz, a maioria dos idosos estudados apresentou algum tipo de dependência nas atividades de banho, vestir e continência, o que corrobora os estudos de Rodrigues (2014), que, ao avaliar a capacidade funcional e os fatores associados em idosos institucionalizados, encontrou a prevalência de tal dependência nesses mesmos itens. Ainda assim, neste estudo, o grupo de idosos que apresentou risco elevado para queda era composto de idosos que apresentavam algum grau de dependência em algum dos itens avaliados pelo índice de Katz. Isso sugere que a diminuição da capacidade funcional contribui para o risco de queda.

Para Rebelatto, Castro e Chan (2007), os idosos longevos mostram-se mais propensos a sofrer queda, pois a massa muscular reduz com o envelhecimento e, quando somada ao aumento da inatividade física, torna o idoso mais suscetível a instabilidades. Dessa forma, a força muscular parece ser um determinante importante do índice de quedas. Nesse contexto, avaliar a força muscular pela FPP permite inferir sobre o risco de queda, já que as pessoas com pouca força nas mãos, geralmente, também apresentam fraqueza nos outros grupos musculares. Esses dados reforçam os achados deste estudo, no qual se identificou grande quantidade de idosos com FPP diminuída.

Dias et al. (2010) apontaram que a força muscular exerce influência sobre as
AVDs, ao passo que, se o idoso tem FM diminuída, ele apresentará diminuição na capacidade funcional. No entanto, neste estudo, a maioria dos idosos apresentaram maior funcionalidade de acordo com a avaliação da $\mathrm{CF}$, por meio do índice de Katz, mesmo com a predominância de FM diminuída. Contudo, a diferença entre a classificação menor e maior funcionalidade foi de apenas um indivíduo. Sugere-se que tal fato tenha ocorrido porque a amostra foi pequena.

Por fim, esses achados evidenciam a importância de profissionais capacitados e familiares/cuidadores orientados quanto à prevenção de queda no ambiente hospitalar. Da mesma forma, destaca-se a necessidade da implementação de sistemas de avaliação do equilíbrio e de programas de exercícios preventivos, visando à diminuição da intensidade de oscilação postural, a fim de minimizar os efeitos fisiológicos do envelhecimento.

Apontamos como limitação deste estudo, o pequeno tamanho amostral, que dificultou a aplicação de análises de correlação.

\section{Conclusão}

De acordo com os resultados obtidos, pode-se concluir que o risco de queda na população investigada mostrou-se de médio a elevado. Observou-se uma porcentagem grande de sedentários e de idosos com força muscular reduzida, o que pode estar associado ao perfil de risco de queda encontrado nessa população. Em relação à caracterização da amostra, observou-se a prevalência do sexo fe- 
minino e de longevos, o que sugere, da mesma forma, um maior risco de queda nessa população. Acreditamos que esse risco mostra-se maior, principalmente, para a população longeva, devido às alterações próprias do envelhecimento, que, associadas às condições do ambiente hospitalar, acabam por comprometer a capacidade funcional do indivíduo, favorecendo o risco para tal evento.

\section{Profile risk of fall in elderly patients hospitalized in a geriatric unit of a university hospital in the city of Porto Alegre}

\section{Abstract}

Falls are a major health problem for the elderly, culminate in loss of functional autonomy and consequently the quality of life. This study aims to observe the risk of fall in elderly patients hospitalized in a geriatric unit, as well as associated factors that influence such an event. This is a cross-sectional descriptive study. In addition to assessing the risk of falling through a scale (Morse Fall Scale - MFS) and a test (Timed Up and Go - TUG), an instrument consisting of demographic data (age and sex) was applied, questioning the practice of prehospital physical activity and evaluation of factors associated with falls as muscle strength (Hand Grip Strenght - HGS) and functional capacity (Katz Index). The study included 33 elderly. Most elderly were female $(70 \%)$, the oldest old (56.7\%) and sedentary (73.3\%). According to the TUG, most elderly was in average risk of falling (56.7\%). According MFS, most elderly found at a high risk of falling $(80 \%)$. Six patients $(19.8 \%)$ had a fall from height. According to the Katz index, most elderly had higher functionality $(36.7 \%)$. According to HGS most elderly showed reduced FPP. According to the results it can be concluded that the risk of falls in this population proved medium to high. It was noted the prevalence of female and oldest, which suggests a higher risk of fall in this population. There was a large percentage of sedentary and elderly with reduced muscle strength, which may be associated with fall of this population risk profile.

Keywords: Accidental falls. Hospitalization. Elderly.

\section{Agradecimentos}

Aos idosos do Hospital São Lucas da Pontifícia Universidade Católica do Rio Grande do Sul (PUCRS) pela colaboração e acolhimento. Às professoras Mara Regina Knorst, Clarissa Netto Blattner, Janete de Souza Urbanetto e Raquel Milani El-kik pela orientação na realização desta pesquisa.

\section{Referências}

ABREU, H. C. A et al. Incidência e fatores preditores de quedas de idosos hospitalizados. Revista de Saúde Pública, Cuiabá, v. 49, n. 37, p. 1-9, ago. 2015.

AL TEHEWY, M. M.; AMIN, G. E.; NASSAR, N. W. A study of rate and predictors of fall among elderly patients in a university hospital. Journal of Patient Safety, Cairo, v. 11, n. 4, p. 210-214, Dec. 2015.

ALVARES, L. M. et al. Ocorrência de quedas em idosos residentes em instituições de longa permanência em Pelotas, Rio Grande do Sul, Brasil. Cadernos de Saúde Pública, Rio de Janeiro, v. 26, n. 1, p. 31-40, jun. 2010. 
BECHARA, F. T.; SANTOS, S. M. S. Efetividadede um programa fisioterapêutico para treino de equilíbrio em idosos. Revista Saúde e Pesquisa, Londrina, v. 1, n. 1, p. 15-20, jan./ abr. 2008.

BOHANNON, R. W. Reference values for adult grip strength measured with a Jamar dynamometer: a descriptive meta-analysis. Physiotherapy, New York, v. 92, n. 1, p. 11-15, Mar. 2006.

BÓS, A. J. G.; KIMURA, A. Y. Internações por condições sensíveis à atenção primária em idosos com 80 anos ou mais em um hospital universitário do Rio Grande do Sul. Revista da AMRIGS, Porto Alegre, v. 59, n. 2, p. 106-111, abr./jun. 2015.

BRITO, T. A. et al. Quedas e capacidade funcional em idosos longevos residentes em comunidade. Texto e Contexto Enfermagem, Florianópolis, v. 22, n. 1, p. 43-51, jan./mar. 2013.

CAMARGOS, M. C. S.; PERPÉTUO, I. H. O.; MACHADO, C. J. Expectativa de vida com incapacidade funcional em idosos em São Paulo, Brasil. Revista Panamericana de Salud Pública, São Paulo, v. 17, n. 6, p. 379-386, jan. 2005.

CRUZ-JENTOFT, A. J. et al. Sarcopenia: European consensus of definition and diagnosis. Age and Ageing. Journal of Applied Physiology, Madrid, v. 39, n. 1, p. 412-423, Apr. 2010.

DIAS, J. A. et al. Força de preensão palmar: métodos de avaliação e fatores que influenciam a medida. Revista Brasileira de Cineantropometria \& Desempenho Humano, Florianópolis, v. 12, n. 3, p. 209-216, nov. 2010.

DUARTE, S. C. M et al. Eventos adversos e segurança na assistência de enfermagem. Revista Brasileira de Enfermagem, Rio de Janeiro, v. 68, n. 1, p. 144-154, jan./fev. 2015. DUARTE, Y. A. O.; ANDRADE, C. L.; LEBRÃO, M. L. O Índex de Katz na avaliação da funcionalidade dos idosos. Revista da Escola de Enfermagem da USP, São Paulo, v. 41, n. 2, p. 317-325, jun. 2007.
DYKES, P. C. et al. Fall prevention in acute care hospitals: a randomized trial. JAMA, Boston, v. 304, n. 17, p. 1912-1918, Nov. 2010. DYKES, P. C et al. Why do patients in acute care hospitals fall? Can falls be prevented? Journal of Nursing Administration, Boston, v. 39, n. 6, p. 299-304, June 2009.

EVANS, D. et al. Fall risk factors in the hospital setting: a systematic review. International Journal of Nursing Practice, Adelaide, v. 7, n. 1, p. 38-45, Feb. 2001.

FERREIRA, D. C. O.; YOSHITOME, A. Y. Prevalência e caraterísticas das quedas de idosos institucionalizados. Revista Brasileira de Enfermagem, Brasília, v. 6, n. 63 p. 991997, nov./dez. 2010.

GUIMARÃES, L. H. C. T. et al. Comparação da propensão de quedas entre idosos que praticam atividade física e idosos sedentários. Revista Neurociências, Lavras, v. 12, n. 2, p. 68-72, abr./jun. 2004.

INSTITUTO BRASILEIRO DE GEOGRAFIA E ESTATÍSTICA. Censo Demográfico 2010. Disponível em: http://www.ibge.gov. br/home/estatistica/populacao/censo2010/ default_resultados_amostra.shtm. Acesso em: 15 ago. 2014.

ISHIZUKA, M. A. et al. Falls by elders with moderate levels of movement functionality. Clinics, São Paulo, v. 60, n. 1, p. 41-46, Jan./ Feb. 2005.

KATZ, S. et al. Studies of illness in the aged. The index of ADL: a standardized measure of biological ans psychosocial function. JAMA, Chicago, v. 185, n. 12, p. 914-919, Sept. 1963.

LIN, C. H. et al. Associated factors for falls among the community-dwelling older people assessed by annual geriatric health examinations. PLoS One, Taipé, v. 6, n. 4, p. 1-5, Apr. 2011.

LIN, M. R. et al. Psychometric comparisons of the timed up and go, one-leg stand, functional reach and Tinetti balance measures in community-dwelling older people. Journal of the American Geriatrics Society, Taipé, v. 52, n. 8, p. 1343-1348, Aug. 2004. 
LOJUDICE, D. C. et al. Quedas de idosos institucionalizados: ocorrência e fatores Associados. Revista Brasileira de Geriatria e Gerontologia, Rio de Janeiro, v. 13, n. 3, p. 403-412, jun. 2010. MACEDO, D. O.; FREITAS, L. M.; SCHEICHER, M. E. Preensão palmar e mobilidade funcional em idosos com diferentes níveis de atividade física. Fisioterapia e Pesquisa, São Paulo, v. 21, n. 2, p. 151-155, jan. 2014.

MACIEL, A. C. C.; GUERRA, R. O. Influência dos fatores biopsicossociais sobre a capacidade funcional de idosos residentes no nordeste do Brasil. Revista Brasileira de Epidemiologia, São Paulo, v. 10, n. 2, p. 178-89, jun. 2007.

MORSE, J. M.; MORSE, R. M.; TYLKO, S. J. Development of a scale to identify the fallprone patient. Canadian Journal on Aging, Canada, v. 8, n. 4, p. 366-377, Jan. 1989.

NASCIMENTO, B. N. et al. Risco para quedas em idosos da comunidade: relação entre tendência referida e susceptibilidade para quedas com o uso do teste clínico de interação sensorial e equilíbrio. Revista Brasileira de Clinica Médica, Santos, v. 7, n. 1, p. 95-99, mar. 2009.

OLIVER, D.; HEALEY, F.; HAINES, T. P. Preventing falls and fall-related injuries in hospitals. Clinincs in Geriatric Medicine, London, v. 26, n. 4, p. 645-92, Nov. 2010.

ORGANIZAÇÃO MUNDIAL DA SAÚDE. Atividade física e idosos: níveis recomendados de atividade física para idosos acima de 65 anos. 2010. Disponível em: <http://www. who.int/dietphysicalactivity/factsheet_olderadults/en/index.html>. Acesso em: 30 set. 2016.

PODSIADLO, D.; RICHARDSON, S. The timed "Up \& Go": a test of basic functional mobility for frail elderly pearsons. Journal of the American Geriatrics Society, Canada, v. 39, n. 2, p. 142-148, Feb. 1991.

REBELATTO, J. R.; CASTRO, A. P.; CHAN, A. Quedas em idosos institucionalizados: características gerais, fatores determinantes e relações com a força de preensão manual. Acta Ortopédica Brasileira, São Paulo, v. 15, n. 3, p. 151-154, jan. 2007.
REIS, M. G. et al. Quedas em idosos com perturbações mentais residentes em unidade de saúde de longa duração. Revista Portuguesa de Enfermagem de Saúde Mental, Porto, v. 1, n. 33, p. 1-5, abr. 2014.

RODRIGUES, D. F. Avaliação funcional e fatores associados em idosos institucionalizados no municipio de Recife-PE. 2014. $68 \mathrm{f}$. Dissertação (Mestrado em Saúde Coletiva) Centro de Ciências da Saúde, Universidade Federal de Pernambuco, Pernambuco, 2014. RODRIGUES, I. G.; FRAGA, G. P.; BARROS, M. B. A. Quedas em idosos: fatores associados em estudo de base populacional. Revista Brasileira de Epidemiologia, Campinas, v. 17, n. 3, p. 705-718, jul./set.2014.

RUBENSTEIN, L. Z. et al. The Sepulveda VA Geriatric Evaluation Unit: data on four-year outcomes and predictors of improved patient outcomes. Journal of the American Geriatrics Society, Los Angeles, v. 32, n. 7, p. 503-512, July 1984.

SALES, M. V. C. et al. Efeitos adversos da internação hospitalar para o idoso. Geriatria \& Gerontologia, São Paulo, v. 4, n. 4, p. 238-246, jan. 2010.

SHUBERT, T. E. Are scores on balance screening tests associated with mobility in older adults? Journal of Geriatric Physical Therapy, State of North Carolina, v. 29, n. 1, p. 33-39, Apr. 2006.

SIQUEIRA, F. V. Prevalência de quedas em idosos e fatores associados. Revista de Saúde Pública, São Paulo, v. 4, n. 5, p. 749-756, Oct. 2007.

URBANETTO, J. S. et al. Morse Fall Scale: tradução e adaptação transcultural para a língua portuguesa. Revista da Escola de Enfermagem da USP, São Paulo, v. 47, n. 3, p. 569-575, jun. 2013.

WELFER, M. Causas de internação em uma unidade Geriátrica de um hospital terciário na Região metropolitana de Porto Alegre / RS. 2007. 57 f. Dissertação (Mestrado em Gerontologia Biomédica) - Instituto de Geriatria e Gerontologia, Pontifícia Universidade Católica do Rio Grande do Sul, Porto Alegre, 2007. 